\title{
Modified Average Sample Number for Improved Double Sampling Plan Based on Truncated Life Test Using Exponentiated Distributions
}

\author{
O. S. Deepa \\ Department of Mathematics, Amrita School of Engineering, Coimbatore Amrita Vishwa Vidyapeetham, India
}

Received April 18, 2020 ; Revised June 30, 2020; Accepted July 20, 2020

(a): [1] O. S. Deepa , "Modified Average Sample Number for Improved Double Sampling Plan Based on Truncated Life Test Using Exponentiated Distributions," Mathematics and Statistics, Vol. 8, No. 5, pp. 542 - 550, 2020. DOI: 10.13189/ms.2020.080507.

(b): O. S. Deepa (2020). Modified Average Sample Number for Improved Double Sampling Plan Based on Truncated Life Test Using Exponentiated Distributions. Mathematics and Statistics, 8(5), 542 - 550. DOI: 10.13189/ms.2020.080507.

Copyright $(2020$ by authors, all rights reserved. Authors agree that this article remains permanently open access under the terms of the Creative Commons Attribution License 4.0 International License

\begin{abstract}
The reliability of the product has developed a dynamic issue in a worldwide business market. Generally acceptance sampling guarantees the superiority of the product. In acceptance sampling plan, increasing the sample size may lead to minimization of customers' risk of accepting bad lots and producers' risk of rejecting good lots to a certain level but will increase the cost of inspection. Hence truncation of life test time may be introduced to reduce the cost of inspection. Modified Average Sample Number (MASN) for Improved Double Sampling Plan (IDSP) based on truncated life test for popular exponentiated family such as exponentiated gamma, exponentiated lomax and exonentiated Weibull distribution are considered. The modified ASN creates a band width for average sample number which is much useful for the consumer and producer. The interval for average sample number makes the choice of consumer with a maximum and minimum sample size which is of much benefit without any loss for the producer. The probability of acceptance and average sample number based on modified double sampling plan for lower and upper limit is computed for the exponentiated family. Optimal parameters of IDSP under various exponentiated families with different shape parameters were computed. The proposed plan is compared over traditional double sampling and modified double sampling using Gamma distribution, Weibull distribution and Birnbaum-Saunders distribution and shows that the proposed plan with respect to exponentiated family performs better than all other plans. The tables were provided for all distributions. Comparative
\end{abstract}

study of tables based on proposed exponentiated family and earlier existing plan are also done.

Keywords Exponentiated Gamma, Exponentiated Lomax, Exponentiated Weibull, Exponentiated Exponential, Average Sample Number, Modified Double Sampling Plan

\section{Introduction}

The main goal of the manufacturing industries is improving the quality of the product which could be attained by acceptance sampling plans. In the acceptance sampling plan, a sample is taken from the lot and the lot is accepted or rejected based on the inspection of the sample from the lot. The number of failures is static and is done based on the opinion of both producer and consumer. The failures that are considered after inspection is more than the static number specified then the lot is rejected. This process is known as life test. Various studies were made on acceptance sampling $[2,3,5,10,11,12,13,14,15,16]$.

The parameters of the modified double sampling plan are $n_{1}, n_{2}, c_{1}$ and $c_{2}$. The probability of acceptance of the MDSP as given by Aslam et al [2]

$$
P_{a}(p)=\sum_{i=1}^{c_{1}}{ }^{n_{1}} C_{i} p^{i}(1-p)^{n_{1}-i}+
$$




$$
+\sum_{j=c_{1}+1}^{c_{2}}{ }_{i}^{n_{1}} C_{j} p^{j}(1-p)^{n_{1}-j} \sum_{i=1}^{c_{1}}{ }^{n_{2}} C_{i} p^{i}(1-p)^{n_{2}-i}
$$

and

$$
A S N(p)=n_{1}+n_{2} \sum_{i=c_{1}+1}^{c_{2}}{ }^{n_{1}} C_{i} p^{i}(1-p)^{n_{1}-i}
$$

Here $p$ is called the probability of failure which can be determined based on the distribution of lifetime of a product. Generally acceptable reliability level $p_{1}$, lot tolerance reliability level $p_{2}$, producer's risk- $\alpha$ consumer's risk $\beta$ are mostly considered.

\section{Methodology}

\subsection{Modified Average Sample Number-MASN}

Generally Minimize ASN subject to $P_{a}\left(p_{1}\right) \geq 1-\alpha$ and $P_{a}\left(p_{2}\right) \leq \beta$ is calculated for the average sample number. The modified ASN creates a band for the consumer's confidence level which does not affect the probability of acceptance. The interval for average sample number makes the choice of consumer with a maximum and minimum sample size which is of much benefit without any loss for the producer. Another advantage is that in spite of different sample size $\left[n_{1}, n_{2}\right]$, the probability of acceptance remains constant and average sample number also remains constant or slightly deviates. It will be better to use $\beta_{1}$ and $\beta_{2}$ instead of $\beta$ which is beneficial for both producers and consumers because various values of $\beta$ with a small difference may make computation lengthy and cannot predict an interval for sample size with a fixed average sample number and probability of acceptance. The choice of any sample size within the interval favours both producers and consumers. So the optimized problem for modified ASN is given as:

$$
\operatorname{MASN}_{L}\left(p_{2}\right)
$$

subject to $P_{a L}\left(p_{1}\right) \geq 1-\alpha \quad, \quad P_{a L}\left(p_{2}\right) \leq \beta_{1} \quad$, $n_{11}>1, n_{21}>1, c_{2}>c_{1}>0$.

and

$$
\text { Minimize } \operatorname{MASN}_{U}\left(p_{2}\right)
$$

subject to $P_{a U}\left(p_{1}\right) \geq 1-\alpha \quad, \quad P_{a U}\left(p_{2}\right) \leq \beta_{2}$ $n_{12}>1, n_{22}>1, c_{2}>c_{1}>0$

where $\beta_{1}-\beta_{2}<\delta$, the threshold $\delta$ can be any value depending on need of the producer and consumer. (for example $\delta=0.001,0.01,0.1$ )

\subsection{Improved Double Sampling Plan: IDSP}

The probability of acceptance of improved plan is given by

$$
\begin{gathered}
P_{a L}(p)=\left[\sum_{i=1}^{c_{1}}{ }^{n_{11}} C_{i} p^{i}(1-p)^{n_{11}-i}+\sum_{j=c_{1}+1}^{c_{2}}{ }_{i}^{n_{11}} C_{j} p^{j}(1-p)^{n_{11}-j} \sum_{i=1}^{c_{1}}{ }^{{ }_{21}} C_{i} p^{i}(1-p)^{n_{21}-i}\right] \\
P_{a U}(p)=\left[\sum_{i=1}^{c_{1}}{ }^{{ }^{12}} C_{i} p^{i}(1-p)^{n_{12}-i}+\sum_{j=c_{1}+1}^{c_{2}}{ }_{i}{ }^{n_{12}} C_{j} p^{j}(1-p)^{n_{12}-j} \sum_{i=1}^{c_{1}}{ }^{{ }_{22}} C_{i} p^{i}(1-p)^{n_{22}-i}\right]
\end{gathered}
$$

$P_{a L}(p)$ and $P_{a U}(p)$ is the probability of acceptance with the sample size $n_{11}, n_{21}, n_{12}$ and $n_{22}$.

The modified average sample number for IMDSP plan is given by

Minimize $A S N_{L}(\alpha)=\left(n_{11}+n_{21}\left(1-P_{a L}\left(p_{\alpha}\right)\right)\right)$, subject to $P_{a L}\left(p_{1}\right) \geq 1-\alpha, P_{a L}\left(p_{2}\right) \leq \beta_{1}$

Minimize $A S N_{U}(\alpha)=\left(n_{12}+n_{22}\left(1-P_{a U}\left(p_{\alpha}\right)\right)\right)$, subject to $P_{a U}\left(p_{1}\right) \geq 1-\alpha, P_{a U}\left(p_{2}\right) \leq \beta_{1}$

Also, $P_{a L}(p)=P_{a U}(p) \geq 1-\alpha$ 


\section{Results}

\subsection{Designing the Improved Double Sampling Plan: \\ IDSP}

The designing of modified double sampling plan is done with respect to three distributions

(i) Exponentiated Gamma Distribution

(ii) Exponentiated Lomax Distribution

(iii) Exponentiated Weibull Distribution

\subsubsection{Exponentiated Gamma Distribution}

The significance of exponentiated gamma distribution lies in its capability to model monotone and non-monotone failure rate functions, which are reasonably widespread in reliability and lifetime data analysis. The cdf and pdf of exponentiated gamma distribution is given by

$$
\begin{gathered}
F(x ; \theta, \lambda)=\left[1-e^{-\lambda x}(\lambda x+1)\right]^{\theta}, \theta, \lambda, x>0 \\
f(x ; \theta, \lambda)=\theta \lambda^{2} x e^{-\lambda x}\left[1-e^{-\lambda x}(\lambda x+1)\right]^{\theta-1}, \theta, \lambda, x>0
\end{gathered}
$$

where $\theta$ is the shape parameter and $\lambda$ is the scale parameter

The mean life time under exponentiated gamma distribution is given by

$$
\theta=\frac{\gamma}{\lambda}\left[2+A_{1}(\gamma)\right]
$$

where

$$
A_{1}(\gamma)=\sum_{j=1}^{\infty} \sum_{k=0}^{j}(-1)^{j}\left(\begin{array}{l}
\gamma-1 \\
j
\end{array}\right)\left(\begin{array}{l}
j \\
k
\end{array}\right) \frac{\Gamma(r+k+2)}{(1+j)^{r+k+2}}, r=1,2 \ldots
$$

According to Asalm and Jun [2] ,the termination life time can be expressed as the multiple of specified average life $\theta_{0}$ and the termination ratio $a$ i.e., $t_{0}=a \theta_{0}$. Hence the cumulative distribution function can be rewritten as

$$
p=\left[1-e^{-\left(\frac{\theta_{0}}{\theta}\right)\left(a \gamma\left(2+A_{1}(\gamma)\right)\right)}\left\{\left(\frac{\theta}{\theta_{0}}\right)(a \gamma)\left(\left(2+A_{1}(\gamma)\right)+1\right\}\right]^{\gamma}\right.
$$

Hence in case of exponentiated gamma distribution, the ARL and the LTRL are attained by considering $\left(\frac{\theta}{\theta_{0}}\right)>1$ and $\left(\frac{\theta}{\theta_{0}}\right)=1$.

These ideal parameters of the plan under exponentiated Gamma distribution are given in Table 1-2 with unequal and equal sample size and shape parameter $\gamma=1$ and $\gamma=2$. It is noted from the Table 1-2 that

(i) For fixed $\beta_{1}$ as $\frac{\theta}{\theta_{0}}$ increases, the value of ASN also increases and the interval of ASN is narrow. For example if $\beta_{1}=0.25$ and $\frac{\theta}{\theta_{0}}=8,10,12$ then the ASN is given by $[6.354,7.926],[6.356,8.711]$, $[6.356,9.104]$

(ii) As $\beta_{1}$ increases, the ASN also increases but the interval of ASN is narrow.

(iii) In Table 1, for $\beta_{1}=0.05$ with $n_{11}=8, n_{21}=9, n_{12}=8, n_{22}=14$ the ASN is [9.362,10.119].

(iv) As the shape parameter increases from $\gamma=1$ to $\gamma=2$, the ASN decreases.

(v) For different values of consumers risk and termination ratios , range of ASN is [6,10], for $c_{1}=0, c_{2}=1$ which is a minimum value compared to all existing plan . 
Table 1. Ideal parameters of specified distribution under exponentiated Gamma distribution with shape parameter $\gamma=2$ and $a=1$.

\begin{tabular}{|c|c|c|c|c|c|c|c|}
\hline$\beta$ & $\frac{\theta}{\theta_{0}}$ & $n_{11}, n_{12}$ & $P_{a L}$ & $A S N_{L}$ & $n_{12}, n_{22}$ & $P_{a U}$ & $A S N_{U}$ \\
\hline & 4 & 4,5 & 0.96959 & 5.963 & 4,9 & 0.95429 & 7.534 \\
\hline \multirow[t]{4}{*}{0.25} & 6 & 4,5 & 0.99258 & 5.963 & 4,10 & 0.98743 & 7.926 \\
\hline & 8 & 4,6 & 0.99703 & 6.354 & 4,11 & 0.99518 & 8.319 \\
\hline & 10 & 4,6 & 0.99871 & 6.356 & 4,12 & 0.99773 & 8.711 \\
\hline & 12 & 4,6 & 0.99936 & 6.356 & 4,13 & 0.99878 & 9.104 \\
\hline \multirow[t]{4}{*}{0.1} & 6 & 6,6 & 0.98592 & 7.552 & 6,10 & 0.98001 & 8.586 \\
\hline & 8 & 6,7 & 0.99447 & 7.810 & 6,11 & 0.9923 & 8.844 \\
\hline & 10 & 6,8 & 0.99734 & 8.069 & 6,12 & 0.9963 & 9.103 \\
\hline & 12 & 6,9 & 0.99854 & 8.327 & 6,12 & 0.99818 & 9.103 \\
\hline \multirow[t]{4}{*}{0.05} & 6 & 8,8 & 0.9756 & 9.211 & 8,11 & 0.9701 & 9.665 \\
\hline & 8 & 8,9 & 0.99053 & 9.362 & 8,13 & 0.98776 & 9.680 \\
\hline & 10 & 8,9 & 0.99584 & 9.362 & 8,14 & 0.99427 & 10.119 \\
\hline & 12 & 8,10 & 0.99774 & 9.514 & 8,15 & 0.99694 & 10.271 \\
\hline \multirow[t]{3}{*}{0.01} & 8 & 12,13 & 0.97999 & 12.5691 & 12,15 & 0.97805 & 12.657 \\
\hline & 10 & 12,14 & 0.9906 & 12.613 & 12,16 & 0.9897 & 12.700 \\
\hline & 12 & 12,15 & 0.99451 & 12.657 & 12,17 & 0.9931 & 12.744 \\
\hline
\end{tabular}

Table 2. Ideal parameters of specified distribution under exponentiated Gamma distribution with shape parameter $\gamma=2$ and $a=2$

\begin{tabular}{|c|c|c|c|c|c|c|c|}
\hline$\beta$ & $\frac{\theta}{\theta_{0}}$ & $n_{11}, n_{12}$ & $P_{a L}$ & $A S N_{L}$ & $n_{12}, n_{22}$ & $P_{a U}$ & $A S N_{U}$ \\
\hline 0.25 & 4 & 3,6 & 0.9953 & 5.3434 & 3,10 & 0.9933 & 6.906 \\
\hline & 6 & 3,7 & 0.9997 & 5.734 & 3,11 & 0.9995 & 7.296 \\
\hline & 8 & 3,8 & 0.99995 & 6.125 & 3,12 & 0.9993 & 7.686 \\
\hline & 10 & 3,9 & & 6.515 & 3,13 & 0.9998 & 8.077 \\
\hline 0.1 & 4 & 4,4 & 0.9954 & 5.087 & 4,7 & 0.99305 & 5.902 \\
\hline & 6 & 4,5 & 0.9996 & 5.359 & 4,8 & 0.99947 & 6.174 \\
\hline & 8 & 4,6 & 0.9999 & 5.630 & 4,9 & 0.99992 & 6.445 \\
\hline & 10 & 4,6 & 0.9999 & 5.630 & 4,9 & 0.99992 & 6.445 \\
\hline 0.05 & 4 & 5,6 & 0.99184 & 6.063 & 5,9 & 0.9890 & 6.594 \\
\hline & 6 & 5,7 & 0.99937 & 6.2404 & 5,9 & 0.99923 & 6.594 \\
\hline & 8 & 5,7 & 0.99992 & 6.2404 & 5,10 & 0.99989 & 6.772 \\
\hline 0.01 & 10 & 5,8 & 0.9998 & 6.418 & 5,12 & 0.99997 & 6.875 \\
\hline & 6 & 8,11 & 0.99792 & 8.4429 & 8,15 & 0.97648 & 8.604 \\
\hline & 8 & 8,12 & 0.99829 & 8.4832 & 8,16 & 0.99788 & 8.644 \\
\hline & 10 & 8,14 & 0.99974 & 8.5637 & 8,18 & 0.999969 & 8.725 \\
\hline
\end{tabular}




\subsubsection{Exponentiated Lomax Distribution}

Two parameter exponentiated Lomax distributions are used in analyzing several lifetime data.

The CDF and PDF of a exponentiated Lomax distribution is given by

$$
\begin{aligned}
& F(x)=\left[1-(1+\lambda x)^{-\theta}\right]^{\alpha}, x>0 ; \theta, \alpha, \lambda>0 \\
& f(x)=\alpha \theta \lambda\left[1-(1+\lambda x)^{-\theta}\right]^{\alpha-1}(1+\lambda x)^{-(\theta+1)}
\end{aligned}
$$

$\alpha, \gamma$ are the shape parameters and $\lambda$ is the scale parameter.

The mean life time under exponentiated exponential distribution is given by

$$
\theta=\frac{\alpha}{\lambda}\left\{\beta\left(1-\frac{1}{\gamma}, \alpha\right)-\beta(1, \alpha)\right\}
$$

According to Asalm and Jun [2], the cumulative distribution function can be rewritten as

$$
p=\left[1-\left(1+\frac{a \alpha}{\left(\frac{\theta}{\theta_{0}}\right)}\left[\beta\left(1-\frac{1}{\gamma}, \alpha\right)-\beta(1-\alpha)\right]\right]^{-\gamma}\right]^{\alpha}
$$

These ideal parameters of the MDSP plan under exponentiated Lomax distribution are given in Table 3-5 with unequal and equal sample size and shape parameter $\gamma=1$ and $\gamma=2$, It is noted from the Table 3-5 that

(i) For fixed $\beta_{1}$ as $\frac{\theta}{\theta_{0}}$ increases, the value of ASN increases and the interval of ASN is very narrow. For example if $\beta_{1}=0.25$ and $\frac{\theta}{\theta_{0}}=6,8,10$ then the ASN is given by $[10.115,10.738],[11.049,11.672]$, $[12.295,13.229]$

(ii) As $\beta_{1}$ increases, the $\mathrm{ASN}$ also increases but the interval of ASN is very narrow.

(iii) In Table 7 , for $\beta_{1}=0.05, \frac{\theta}{\theta_{0}}=10$ with $n_{11}=6, n_{21}=8, n_{12}=6, n_{22}=10$ the $\mathrm{ASN}$ is 7 .

(iv) As the termination ratio increases from $a=0.5$ to $a=1$, the ASN also increases.

(v) For different values of consumers risk, the mean ratio values and termination ratios the maximum value of ASN is only 23 which is a minimum value compared to all existing plan.

Table 3. Ideal parameters of specified distribution under exponentiated Lomax distribution with shape parameter $\gamma=1$ and $a=1$

\begin{tabular}{|c|c|c|c|c|c|c|c|}
\hline$\beta$ & $\frac{\theta}{\theta_{0}}$ & $n_{11}, n_{12}$ & $P_{a L}$ & $A S N_{L}$ & $n_{12}, n_{22}$ & $P_{a U}$ & $A S N_{U}$ \\
\hline 0.25 & 14 & 3,3 & 0.97044 & 4.125 & 3,3 & 0.9547 & 4.125 \\
\hline & 16 & 3,3 & 0.9547 & 4.125 & 3,4 & 0.9564 & 4.5 \\
\hline & 18 & 3,3 & 0.96402 & 4.5 & 3,5 & 0.95841 & 4.875 \\
\hline & 20 & 3,4 & 0.964421 & 4.5 & 3,5 & 0.96536 & 4.875 \\
\hline 0.1 & 20 & 4,4 & 0.9587 & 5 & 4,5 & 0.95162 & 5.25 \\
\hline & 22 & 4,5 & 0.95897 & 5.25 & 4,5 & 0.95897 & 5.25 \\
\hline 0.05 & 24 & 4,6 & 0.96015 & 5.5 & 4,7 & 0.95572 & 5.75 \\
\hline & 28 & 5,6 & 0.955004 & 5.937 & 5,7 & 0.950301 & 6.09 \\
\hline & 30 & 5,6 & 0.960461 & 6.01 & 5,7 & 0.956275 & 6.09 \\
\hline 0.01 & 36 & 5,7 & 0.961236 & 6.09 & 5,8 & 0.957609 & 6.25 \\
\hline & 38 & 7,8 & 0.95439 & 7.437 & 7,9 & 0.95091 & 7.492 \\
\hline
\end{tabular}


Table 4. Ideal parameters of specified distribution under exponentiated Lomax distribution with shape parameter $\gamma=2$ and $a=1$

\begin{tabular}{|c|c|c|c|c|c|c|c|}
\hline$\beta$ & $\frac{\theta}{\theta_{0}}$ & $n_{11}, n_{12}$ & $P_{a L}$ & $A S N_{L}$ & $n_{12}, n_{22}$ & $P_{a U}$ & $A S N_{U}$ \\
\hline 0.25 & 6 & 6,7 & 0.97924 & 8.4917 & 6,8 & 9.88476 & 8.8476 \\
\hline & 8 & 7,10 & 0.99544 & 10.1146 & 7,12 & 9.96255 & 10.7375 \\
\hline & 10 & 7,13 & 0.99297 & 11.0490 & 7,15 & 9.992162 & 11.6719 \\
\hline & 12 & 7,17 & 0.99544 & 12.2948 & 7,20 & 9.99423 & 13.2292 \\
\hline 0.1 & 6 & 9,10 & 0.95734 & 11.2525 & 9,12 & 0.95223 & 11.7030 \\
\hline & 8 & 9,13 & 0.979818 & 11.9283 & 9,15 & 0.977718 & 12.3788 \\
\hline & 10 & 9,14 & 0.98949 & 12.15336 & 9,15 & 0.98762 & 12.3788 \\
\hline & 12 & 9,15 & 0.994173 & 12.6040 & 9,18 & 0.993628 & 13.0545 \\
\hline 0.05 & 8 & 11,16 & 0.970596 & 13.4777 & 11,18 & 0.96818 & 13.7875 \\
\hline & 10 & 11,20 & 0.9836 & 14.0972 & 11,22 & 0.98243 & 14.4069 \\
\hline & 12 & 11,25 & 0.980749 & 14.8715 & 11,27 & 0.98906 & 15.1812 \\
\hline 0.01 & 10 & 17,30 & 0.96433 & 18.2778 & 18,30 & 0.961958 & 19.01478 \\
\hline & 12 & 20,30 & 0.97656 & 20.7399 & 20,35 & 0.973073 & 20.63424 \\
\hline & 14 & 22,33 & 0.983526 & $22 . .4316$ & 22,42 & 0.980506 & 22.5494 \\
\hline
\end{tabular}

Table 5. Ideal parameters of specified distribution under exponentiated Lomax distribution with shape parameter $\gamma=2$ and $a=2$

\begin{tabular}{|c|c|c|c|c|c|c|c|}
\hline$\beta$ & $\frac{\theta}{\theta_{0}}$ & $n_{11}, n_{12}$ & $P_{a L}$ & $A S N_{L}$ & $n_{12}, n_{22}$ & $P_{a U}$ & $A S N_{U}$ \\
\hline 0.25 & 6 & 3,4 & 0.951275 & 4.646 & 3,8 & 0.92231 & 6.292 \\
\hline & 8 & 3,5 & 0.97491 & 5.058 & 3,9 & 0.96132 & 6.704 \\
\hline & 10 & 3,6 & 0.98739 & 5.078 & 3,10 & 0.97839 & 7.115 \\
\hline 0.1 & 8 & 4,6 & 0.96015 & 5.829 & 4,8 & 0.95146 & 6.439 \\
\hline & 10 & 4,7 & 0.97726 & 6.134 & 4,9 & 0.97267 & 6.743 \\
\hline 0.05 & 10 & 4,8 & 0.98589 & 6.439 & 4,11 & 0.08199 & 7.353 \\
\hline & 12 & 6,8 & 0.9600 & 7.129 & 6,10 & 0.9537 & 7.411 \\
\hline 0.01 & 12 & 8,9 & 0.9753 & 7.270 & 6,11 & 0.97167 & 7.5573 \\
\hline
\end{tabular}

\subsubsection{Exponentiated Weibull Distribution:}

The distribution function and the probability density function are given by

$$
G_{\alpha}(x)=\left\{1-\exp \left(-(\lambda x)^{\gamma}\right\}^{\alpha}, x>0, \alpha, \lambda, \gamma>0\right.
$$

$g(x)=\alpha \gamma \lambda^{\gamma} x^{-1}\left\{1-\exp \left(-(\lambda x)^{\gamma}\right\}^{\alpha-1} \exp \left\{-(\lambda x)^{\gamma}\right\}, x>0\right.$

$\alpha, \gamma$ are the shape parameters and $\lambda$ is the scale parameter.

The mean life time under exponentiated weibull distribution is

$$
E(Z)=\left\{\begin{array}{l}
\alpha \lambda^{-1} \Gamma\left(\frac{1}{\gamma}+1\right) \sum_{i=0}^{\alpha-1}\left(\begin{array}{l}
\alpha-1 \\
i
\end{array}\right)(-1)^{i}(i+1)^{-\frac{1}{\gamma}-1}, \alpha \in N \\
\alpha \lambda^{-1} \Gamma\left(\frac{1}{\gamma}+1\right) \sum_{i=0}^{\alpha-1} \frac{\alpha-1}{i !} P_{i}(-1)^{i}(i+1)^{-\frac{1}{\gamma}-1}, \alpha \notin N
\end{array}\right.
$$

According to Asalm and Jun [2], the cumulative distribution function can be rewritten as

$$
p=\left[1-\exp \left\{-\frac{a \theta_{0}}{\theta} \alpha \Gamma\left(\frac{1}{\gamma}+1\right) \sum^{\alpha-1} C_{i}(-1)^{i}(i+1)^{-\frac{1}{\gamma}}-1\right\}\right]^{\alpha}, \alpha \in N
$$


These ideal parameters of the MDSP plan under exponentiated Lomax distribution are given in Table 6-7 with unequal and equal sample size and shape parameter $\gamma=1$ and. It is noted from the Table 6-7 that

(i) For fixed $\beta_{1}$ as $\frac{\theta}{\theta_{0}}$ increases, the value of ASN increases and the interval of ASN is very narrow. For example if $\beta_{1}=0.25$ and $\frac{\theta}{\theta_{0}}=10,12,14$ then the ASN is given by $[2.9691,3.4537],[2.5289,3.6612]$, $[2.6612,4.9075]$ (ii) As $\beta_{1}$ increases, the ASN also increases but the interval of ASN is very narrow.

(iii) In Table 7, for, $\frac{\theta}{\theta_{0}}=10 \quad$ with $n_{11}=2, n_{21}=2, n_{12}=2, n_{22}=13$ the ASN is 3.

(iv) As the termination ratio increases from $a=0.5$ to $a=1$, the ASN also increases.

(v) For different values of consumers' risk, the mean ratio values and termination ratios the maximum value of ASN is only 5 which is a minimum value compared to all existing plan.

Table 6. Optimal parameters of MDSP under exponentiated weibull distribution with shape parameter $\gamma=1$ and $a=1$

\begin{tabular}{|c|c|c|c|c|c|c|c|}
\hline$\beta$ & $\frac{\theta}{\theta_{0}}$ & $n_{11}, n_{12}$ & $P_{a L}$ & $A S N_{L}$ & $n_{12}, n_{22}$ & $P_{a U}$ & $A S N_{U}$ \\
\hline 0.1 & 10 & 2,2 & 0.95973 & 2.9301 & 2,3 & 0.96106 & 3.395 \\
\hline & 12 & 2,3 & 0.96377 & 3.3952 & 2,4 & 0.9519 & 3.8603 \\
\hline & 14 & 2,4 & 0.96334 & 4.283 & 3,6 & 0.95043 & 4.5398 \\
\hline 0.1 & 18 & 3,5 & 0.95637 & 4.283 & 3,6 & 0.95043 & 4.53598 \\
\hline 0.05 & 20 & 3,7 & 0.954 & 4.7968 & 3,8 & 0.95011 & 5.05315 \\
\hline & 22 & 4,4 & 0.9562 & 4.5035 & 4,4 & 0.9562 & 4.5035 \\
\hline 0.01 & 24 & 4,4 & 0.96306 & 4.5001 & 4,5 & 0.95731 & 4.629 \\
\hline
\end{tabular}

Table 7. Optimal parameters of MDSP under exponentiated weibull distribution with shape parameter $\gamma=2$ and $a=1$

\begin{tabular}{|c|c|c|c|c|c|c|c|}
\hline$\beta$ & $\frac{\theta}{\theta_{0}}$ & $n_{11}, n_{12}$ & $P_{a L}$ & $A S N_{L}$ & $n_{12}, n_{22}$ & $P_{a U}$ & $A S N_{U}$ \\
\hline 0.25 & 10 & 2,2 & 0.9675 & 2.9691 & 2,3 & 0.95657 & 3.4537 \\
\hline & 12 & 2,4 & 0.96111 & 2.5289 & 2,5 & 0.9541 & 3.6612 \\
\hline & 14 & 2,5 & 0.96499 & 2.6612 & 2,6 & 0.95985 & 4.9075 \\
\hline 0.1 & 16 & 3,5 & 0.9566 & 4.4981 & 3,6 & 0.95069 & 4.7977 \\
\hline & 18 & 3,7 & 0.9648 & 4.498 & 3,6 & 0.95989 & 4.7977 \\
\hline 0.05 & 20 & 4,6 & 0.95393 & 4.988 & 4,7 & 0.95012 & 5.15274 \\
\hline & 22 & 4,7 & 0.95674 & 4.988 & 4,7 & 0.95001 & 5.15274 \\
\hline
\end{tabular}


Table 8. Comparison of the existing models and the proposed model

\begin{tabular}{|c|c|c|c|c|c|c|c|}
\hline$\beta$ & $\frac{\theta}{\theta_{0}}$ & \multicolumn{7}{|c|}{$A S N\left(p_{2}\right)$ with $\gamma=2$ and $a=1$} \\
\hline & & $\begin{array}{c}\text { Gamma } \\
\text { Distribution }\end{array}$ & $\begin{array}{c}\text { Weibull } \\
\text { Distribution }\end{array}$ & $\begin{array}{c}\text { Birnbaum-Saunders } \\
\text { Distribution }\end{array}$ & $\begin{array}{c}\text { Exponentiated } \\
\text { Gamma } \\
\text { Distribution }\end{array}$ & $\begin{array}{c}\text { Exponentiated } \\
\text { Lomax } \\
\text { Distribution }\end{array}$ & $\begin{array}{c}\text { Exponentiated } \\
\text { Weibull } \\
\text { Distribution }\end{array}$ \\
\hline 0.25 & 4 & $(2,3) 3.447$ & $(2,4) 3.984$ & $(9,10) 11.580$ & & & \\
\hline & 6 & $(2,3) 3.447$ & $(2,12) 7.953$ & $(6,7) 8.219$ & & $(6,7) 8.4917$ & $(7,10) 10.114$ \\
\hline \\
\hline & 10 & $2,8) 5.859$ & $(2,43) 23.332$ & $(5,6) 9.014$ & & $(7,17) 12.294$ & $(2,2) 2.9291$ \\
\hline
\end{tabular}

\section{Discussion}

Modified double sampling plan was done with respect to various distributions and the comparison of existing models and proposed model is shown in Table 8 .

Exponentiated Lomax distribution is found to be

(i) better than Weibull distribution as the ASN value is smaller

(ii) better than Gamma distribution because in Gamma distribution there is jump in ASN values from 3.447 to 5.859 and then to 11.16 where as in EL distribution there is slow change in ASN values. Hence as $\frac{\theta}{\theta_{0}}$ increases the ASN of EL will be smaller.

(iii) better than Birnbaum-Saunders distribution because as $\frac{\theta}{\theta_{0}}$ increases the ASN may not exist.

Exponentiated Weibull Distribution is better than Gamma, Weibull and and Birnbaum-Saunders distribution because the ASN is very smaller for larger values of $\frac{\theta}{\theta_{0}}$.

Exponentiated Gamma Distribution gives smaller ASN values as shape parameter and termination ratio increases.

\section{Exponentiated Exponential Distribution}

It is a particular case of exponentiated weibull distribution.

$$
\begin{gathered}
f(x, \alpha, \lambda)=\alpha \lambda\left(1-e^{-\lambda x}\right)^{\alpha-1} e^{-\lambda x}, x>0 \\
F(x, \alpha, \lambda)=\left(1-e^{-\lambda x}\right)^{\alpha} ; x>0
\end{gathered}
$$

The mean life time under exponentiated exponential distribution is given by

$$
\mu=\frac{1}{\lambda}(\psi(\alpha+1)-\psi(1))
$$

Here $\alpha, \lambda$ are the shape and scale parameters respectively.

The ASN values of Exponentiated Exponential distribution did not give a better result as compared to the proposed model.

\section{Conclusions}

Modified average sample number (MASN) for improved double sampling plan-IDSP based on truncated life test for popular exponentiated family such as exponentiated gamma, exponentiated lomax and exonentiated Weibull distribution were developed and compared. Exponentiated Gamma, Exponentiated Lomax and Exponentiated Weibull perform better than the existing model when shape parameter and mean termination ratio increases. Also, modified average sample number provides an interval which would be much useful for both producer and consumer regarding the sample size and average sample number even when value of the shape parameter and termination ratio increases. Exponentiated distributions performed well compared to other distributions and is better suited to truncated life test in acceptance sampling plan.

\section{REFERENCES}

[1] Aslam M, S. Balamurali, Chi-Hyuck and Aneela-Meer: Time - truncated attribute sampling plans using EWMA for Weibull and Burr type $\mathrm{X}$ distributions, Communication in Statistics - Simulation and Computation 2017

[2] Aslam M, S.Balamurali and Touqeer Arif Improved double acceptance sampling plan based on truncated life test for some popular statistical distributions. 2016, Journal of statistical Computation and simulation

[3] Aslam M, Jun C-H. A double acceptance sampling plan for generalized log-logistic distributions with known shape parameters. J Appl Stat. 2010;37(3):405-414 
[4] Aslam M, Jun C-H, Ahmad M. New acceptance sampling plans based on life tests for Birnbaum-Saunders distributions. J Statist Simul Comput. 2011;81(4):461-470.

[5] Aydemir E, Olgun MO. An application of single and double acceptance sampling plans for manufacturing system. J Eng Sci Design. 2010;2(1):65-71.

[6] Birnbaum ZW, Saunders SC. A new family of life distributions. J. Appl. Probab. 1969;6:637-652.

[7] Deepa O.S. (2015): Application of acceptance sampling plan in green design and manufacturing in the International Journal of Applied Engineering Research ,Special issue, Vol 10, No.2, pp. 1498-1499.

[8] Deepa O.S. (2015): Optimal production policy for the design of green supply chain model in the International Journal of Applied Engineering Research, Special issue,, Vol 10, No.2, 2015, pp. 1600-1601.

[9] Deros BM,Peng CY, Ab Rahaman MN,Ismail AR,Sulong AB: Assessing acceptance sampling application in manufacturing electrical and electronic products. J Achievements Mater Manuf Eng. 2008;31(2):622-628

[10] Kantam RRL, Rosaiah K. Half logistic distribution in acceptance sampling based on life tests. IAPQR Trans. 1998;23:117-125
[11] Purkar S, Maheshwari G, Khandwawala AI. Design of a double acceptance sampling plan to minimize a consumer's risk considering an OC curve; a case study. Int J Emerg Technol. 2011;2(1):114-118.

[12] Shruthi. G and O.S.Deepa, 2018: “Average run length for exponentiated distribution under truncated life test, International Journal of Mechanical Engineering and Technology (IJMET)", Volume 9, Issue 6, pp.1180-1188.

[13] Sreeja M. Krishnan and O. S. Deepa, 2019: Control Charts for Multiple Dependent State Repetitive Sampling Plan Using Fuzzy Poisson Distribution, International Journal of Civil Engineering and Technology (IJCIET), Volume 10, Issue 1, pp.509-519.

[14] S.Balamurali, P. Jaydurga and M. Usha: Optimal design of repetitive group sampling plans for Weibull and gamma distributions with applications and comparisons to the Birnbaum-Saunders distribution Jan 2018, Journal of Applied Statistics

[15] S.Balamurali, P. Jaydurga and M. Usha Designing of Bayesian Multiple Deferred State Sampling plan Based on Gamma- Poisson Distribution, American Journal of Mathematical and Management Sciences, 2016.

[16] Tsai, T. -R. and Wu, S. -J. (2006). Acceptance sampling based on truncated life tests for generalized Rayleigh distribution, Journal of Applied Statistics, 33, 595-600. 